\title{
Chromosome-Scale Genome Sequence of Alternaria alternata Causing Alternaria Brown Spot of Citrus
}

\author{
Yunpeng Gai, ${ }^{1,2}$ Haijie Ma, ${ }^{1,3}$ Yanan Chen, ${ }^{1}$ Lei Li, ${ }^{1}$ Yingzi Cao, ${ }^{1}$ Mingshuang Wang, ${ }^{1,4}$ Xuepeng Sun, ${ }^{1,5}$ \\ Chen Jiao, ${ }^{1,5}$ Brendan K. Riely, ${ }^{2}$ and Hongye $\mathrm{Li}^{1, \dagger}$ \\ ${ }^{1}$ Key Lab of Molecular Biology of Crop Pathogens and Insects, Ministry of Agriculture, Institute of Biotechnology, Zhejiang \\ University, Hangzhou 310058, China \\ ${ }^{2}$ Department of Plant Pathology, University of California, Davis, CA 95616, U.S.A. \\ ${ }^{3}$ School of Agriculture and Food Sciences, Zhejiang Agriculture \& Forestry University, Hangzhou 311300, China \\ ${ }^{4}$ College of Life and Environmental Sciences, Hangzhou Normal University, Hangzhou 310036, China \\ ${ }^{5}$ Boyce Thompson Institute, Cornell University, Ithaca, NY 14853, U.S.A.
}

Accepted 9 March 2021.

\begin{abstract}
Alternaria brown spot (ABS), caused by Alternaria alternata, is an economically important fungal disease of citrus worldwide. The ABS pathogen A. alternata tangerine pathotype can produce a host-specific ACT toxin, which is regulated by ACT toxin gene cluster located in the conditionally dispensable chromosome (CDC). Previously, we have assembled a draft genome of $A$. alternata tangerine pathotype strain $\mathrm{Z} 7$, which comprises 165 contigs. In this study, we report a chromosome-level genome assembly of $A$. alternata $\mathrm{Z7}$ through the combination of Oxford Nanopore sequencing and Illumina sequencing technologies. The assembly of $A$. alternata $\mathrm{Z7}$ had a total size of $34.28 \mathrm{Mb}$, with a GC content of $51.01 \%$ and contig $\mathbf{N}_{50}$ of 3.08 $\mathrm{Mb}$. The genome is encompassed 12,067 protein-coding genes, 34 ribosomal RNAs, and 107 transfer RNAs. Interestingly, A. alternata $\mathrm{Z7}$ is composed of 10 essential chromosomes and 2 CDCs, which is consistent with the experimental evidences of pulsed-field gel electrophoresis. To our best knowledge, this is the first chromosome-level genome assembly of $A$. alternata. In addition, a database for citrus-related Alternaria genomes has been established to provide public resources for the sequences, annotation and comparative genomics data of Alternaria spp. The improved genome sequence and annotation at the chromosome level is a significant step toward a better understanding of the pathogenicity of $A$. alternata. The database will be updated regularly whenever the genomes of newly isolated Alternaria spp. are available. The citrus-related
\end{abstract}

${ }^{\dagger}$ Corresponding author: H. Li; hyli@zju.edu.cn

Funding: This study was supported by the National Natural Science Foundation of China (grants 32001847 and 32072362 ), National Key Research and Development Program of China (grant 2017YFD0202000), and Chinese Modern Agricultural Technology Systems (CARS-26). This work was also supported by the Chinese Scholarship Council (CSC) through an international cooperation project. Y. Gai received a fellowship of Chinese Scholarship Council to do research in the Department of Plant Pathology, University of California, Davis for 20 months.

The author(s) declare no conflict of interest.

(c) (1) () $\odot$ Copyright $(92021$ The Author(s). This is an open access article distributed under the CC BY-NC-ND 4.0 International license.
Alternaria genomes database is open accessible through the Citrus Fungal Disease Database.

Keywords: Alternaria alternata, Alternaria brown spot, fungal pathogen, microbial genome sequencing

The Alternaria alternata species group (Alternaria section Alternaria; Alternaria alternaria (Fr.) Keissl.) consists of economically important filamentous fungi with a wide host range (Tsuge et al. 2013; Woudenberg et al. 2015). A. alternata is composed of at least 11 pathotypes, each of which can produce a unique hostselective toxin that kills host cells before invasion and extensively absorbs nutrients from dead tissues (Akimitsu et al. 2014; Thomma 2003). In citrus plants, there are two distinct pathotypes: (i) A. alternata tangerine pathotype, which produces a hostspecific Alternaria citri toxin (ACT toxin), causing Alternaria brown spot (ABS) of tangerine (Citrus reticulata Blanco), grapefruit (C. paradisi Macfad.), hybrids of tangerine and grapefruit, and hybrids of tangerine and sweet orange (Chung 2012; Huang et al. 2015); and (ii) A. alternata rough lemon pathotype, which produces $A$. citri rough lemon (ACRL toxin), causing leaf spot of rough lemon and Rangpur lime (Tsuge et al. 2013). ABS mainly infects young leaves, young shoots, and young fruit of susceptible citrus, causing small, circular to oval, light-brown to dark-brown necrotic lesions with an obvious yellow halo around the lesion of diseased citrus leaves (Huang et al. 2015; Wang et al. 2010). Interestingly, the production of ACT toxin is regulated by several ACTT genes (ACTT1, ACTT2, ACTT3, ACTT5, ACTT6, ACTTS2, and ACTTS3) located in the conditionally dispensable chromosome (CDC) (Hatta et al. 2002; Wang et al. 2019). Genetic inactivation of ACTT gene will block the biosynthesis of ACT toxin, leading to complete loss of virulence (Ajiro et al. 2010; Miyamoto et al. 2010).

Citrus is the largest subtropical and tropical fruit crop in the world and is cultivated on over 9.28 million ha, with an annual production of 148 million metric tons. The necrotroph A. alternata is an important citrus fungal pathogen with global distribution. In 1903, ABS was first reported in Australia on Emperor mandarin (Cobb 1903). Because of its morphological similarity to the pathogen of Citrus black rot, the causal agent of Alternaria brown rot was 
initially identified as Alternaria citri Ellis \& Pierce. However, A. citri was found to be misidentified in 1983 and was reclassified as A. alternata based on the morphological characteristics (Nishimura and Kohmoto 1983). To date, ABS has been reported in the United States (Whiteside 1976), Israel (Solel 1991), Turkey (Canihos et al. 1997), South Africa (Swart et al. 1998), Spain (Vicent et al. 2000), Brazil, Argentina (Peres et al. 2003), Greece (Elena 2006), Peru (Marín et al. 2006), Colombia, Iran (Kakvan et al. 2012), and Italy (Aiello et al. 2020), and poses a serious threat to the citrus industry. The pathogen A. alternata is able to overwinter in plant residues and soils in the citrus orchard. The spots gradually become round or irregular and are uniformly distributed on the citrus leaves, causing severe defoliation and leaf chlorosis. After defoliation, the hypha in the fallen leaves can produce a large number of conidia, which are widely spread by air currents or rain splashes, and continue to cause further infections (Huang et al. 2015; Li et al. 2015). China is one of the largest citrus-producing and -consuming countries in the world, with a cultivated area of 2.49 million ha and an annual production of 41.38 million metric tons in 2018. ABS caused by A. alternata on citrus was first observed in Wenshan, Yunnan Province, southern China in 2010 (Wang et al. 2010). Subsequently, ABS was observed in Chongqing, Zhejiang, Hunan, Yunnan, Guangdong, Guangxi and Sichuan Provinces, causing serious losses in citrus production (Huang et al. 2015). Without proper management, ABS infection can lead to serious leaf spots, early leaf defoliation, shoot dieback, and early fruit drop. The management of ABS in China, the largest citrus-producing country worldwide, relies heavily on the use of fungicide ( $\mathrm{Li}$ et al. 2015). However, the extensive use of chemical agents not only causes serious pesticide residues in citrus but also potentially threatens human health, and also causes serious ecological and environmental safety problems ( $\mathrm{Li}$ et al. 2015).

The use of long reads derived from high-throughput sequencing is a powerful approach to produce genome assembly of fungal genomes. To date, the genomes of various Alternaria spp., including A. alternata, have been sequenced in the past few years. The available draft genome of $A$. alternata includes $A$. alternata apple pathotype FERA1166 and A. alternata Japanese Pear pathotype FERA650 (Armitage et al. 2020; Dang et al. 2015). These genome sequences indeed provide new insight into the species classification, gene biological function, molecular identification, and evolution of Alternaria spp. However, most of these genome assemblies are highly fragmented because they were generated using shortread sequencing technologies alone or combined with the PacBio long reads. Therefore, it is difficult to distinguish genomic fragments from essential chromosomes (ECs) and CDCs. Previously, we have assembled a draft genome of A. alternata tangerine pathotype Z7, which contains 165 contigs (GenBank accession number GCA_001572055.1) (Wang et al. 2016, 2019). This assembly provides evidence on the identity of $\mathrm{Z7}$ in terms of taxonomical classification (Huang et al. 2015) and has guided multiple biological studies in our lab to address important questions regarding the virulence and regulation of ACT production of Z7 (Fu et al. 2020; Gai et al. 2021; Ma et al. 2019; Wang et al. 2018).

The fungus was grown on potato dextrose broth (PDB) medium ( $200 \mathrm{~g}$ of potato, $20 \mathrm{~g}$ of glucose, and 1 liter of purified water) on a rotary shaker set at $180 \mathrm{rpm}$ at $25^{\circ} \mathrm{C}$. For DNA extraction, the mycelia produced in PDB was filtered and ground into fine powder with liquid nitrogen and stored at $-80^{\circ} \mathrm{C}$. High molecular weight genomic DNA was extracted from 3-day-old Z7 mycelia using the cetyltrimethylammonium bromide method. Illumina HiSeq and Oxford Nanopore technologies were used to generate $7.87 \mathrm{~Gb}$ of paired-end short sequence reads and $13.28 \mathrm{~Gb}$ of long reads, respectively. The 1627,160 Oxford Nanopore reads (386x coverage) have a read length $\mathrm{N}_{50}$ of $28,902 \mathrm{bp}$ after filtering. We assembled the Z7 genome from Oxford Nanopore reads using the assembler Nanopore Error Correction and Assembly Tool (NECAT) with default parameters and polished the assembly with Illumina PE reads using Pilon v1.23 (Walker et al. 2014). The final Z7 assembly included 13 contigs with a total genome size of $34,280,341 \mathrm{bp}$ (Table 1). Benchmarking universal single-copy ortholog (BUSCO) v5.0 $\beta$ with the 'fungi_odb10' library was used to evaluate the completeness of genome assembly (Simão et al. 2015). Results showed that the genome completeness (BUSCO complete + partial) of A. alternata strain Z7 was $97.9 \%$. Other statistics of the genome of A. alternata strain $\mathrm{Z7}$ are shown in Tables 1 and 2.

The transcriptome profiling was performed for A. alternata Z7 and the AaMetR disrupted mutant (Gai et al. 2019). After trimming low-quality sequences, the cleaned RNA-sequencing (RNA-seq) reads were mapped to the reference genome of A. alternata Z7 with hisat2 (version 2.1.0) (Kim et al. 2019), using the following settings: '-p 42 -min-intronlen 20 -max-intronlen 4000'. The resulting alignments were converted into the binary format with SAMtools ( $\mathrm{Li}$ et al. 2009), and the binary alignment files were converted to hint files with bam2hints (with the parameter '-introns only') from AUGUSTUS (version 3.3.0) (Hoff and Stanke 2019). De novo assembly of the transcriptome was performed with the cleaned RNA-seq reads by rnaSPAdes v3.13 (Bushmanova et al. 2019) and Trinity v2.11.0 (Grabherr et al. 2011) with default parameters. For gene prediction and annotation, the genomes were first annotated using a Perl script gmes_petap.pl from the GeneMark-ET v4.33 (Brina et al. 2020), with the RNAseq files as biological evidence. The resulting general feature format (gff) files were used for the protein model training of AUGUSTUS v3.3 (Hoff and Stanke 2019). In addition to GeneMark-ET and AUGUSTUS, the genomes were also annotated using SNAP v2013.02.16. For redundant transcripts and alternative splicing prediction, the transcript reads were mapped to the reference genome by a Perl script Launch_PASA_pipeline.pl from the Program to Assemble Spliced Alignments (PASA) v2.2.0 annotation pipeline (Campbell et al. 2006). For gene prediction by homolog protein, the genomes were annotated by a Perl script homolog_genewise from the GeneWise v2.4.1 (Birney et al. 2004). The software RepeatMasker v4.0.5 was used to screen DNA sequences for interspersed repeats and low complexity DNA sequences. RepeatModeler v1.0.8 was used to identify the transposable elements families. The genome annotation pipeline MAKER (version 2.31.9) (Cantarel et al. 2008) was used to perform genome annotation, with the transcripts as expressed sequence tag

Table 1. Summary of the genome assembly and annotation statistics of Alternaria alternata Z7 (GenBank accession GCA_014751505.1) compared with a previous assembly of Z7 (GenBank accession GCA_001572055.1) and A. solani CBS_143772 (GenBank accession GCA_001572055.1) ${ }^{\mathrm{a}}$

\begin{tabular}{llll}
\hline Strains & $\begin{array}{c}\text { Z7 } \\
\text { (this study) }\end{array}$ & $\begin{array}{c}\text { Z7 } \\
\text { (GCA_001572055.1) }\end{array}$ & $\begin{array}{c}\text { A. solani } \\
\text { altNL03003 }\end{array}$ \\
\hline $\begin{array}{l}\text { Number of contigs } \\
\text { Number of }\end{array}$ & 12 & 165 & 10 \\
$\quad$ chromosomes & 12 & NA & 10 \\
Number of CDC & 2 & NA & 0 \\
Genome size (Mb) & 34.35 & 34.35 & 32.77 \\
GC content (\%) & 50.99 & 50.98 & 51.32 \\
Contig N50 (bp) & $3,085,453$ & $1,128,165$ & $2,866,555$ \\
Contig N90 (bp) & $2,401,432$ & 184,938 & $2,309,181$ \\
Number of & 12,067 & 12,048 & NA \\
$\quad$ protein-coding & & & 97.9 \\
genes & 97.9 & 98.1 & \\
Genome & & \\
$\quad$ completeness $(\%)$ & b & &
\end{tabular}


evidence, the homolog proteins as homology evidence, and the transposable elements as repeat masking. Consequently, gene predictions generated by different software were fed to EVidenceModeler version 1.1.1 to select the best models (Haas et al. 2008), which identified 12,067 protein-coding genes, 34 ribosomal RNAs, and 107 transfer RNAs (Tables 1 and 2).

Carbohydrate-active enzymes (CAZYmes) play a vital role in destroying host cell wall components to establish a successful infection process. The dbCAN v6.0 database (Lombard et al. 2014) was used to predict CAZYmes by using hmmscan, and showed 820 CAZYmes (6.80\% of the proteome), which included 294 glycosyl hydrolases, 155 carbohydrate esterases, 110 glycoside transferases, 75 carbohydrate-binding modules, 161 auxiliary activities, and 25 polysaccharide lyases. The UniProtKB/SwissProt database is a high-quality annotated and nonredundant protein sequence database, which brings together experimental results, computed features, and scientific conclusions. The proteins were functionally annotated by using Protein-Protein BLAST v2.6.0 against the Swiss-Prot database (Bairoch and Apweiler 2000) with the parameters '-evalue 1e-5 -max_target_seqs 20', which resulted in a best-hit description for 7,907 genes representing $65.52 \%$ of the proteome. The pathogenicity genes were predicted by using BLASTP against the Pathogen-Host Interaction (PHI) database with a strict threshold of Match $\geq 50 \%$ and Identity $\geq$ $50 \%$ (Winnenburg et al. 2006), which identified 883 putative PHI genes (7.31\% of the proteome). SignalP v5.0 analysis of the proteomes of $A$. alternata $\mathrm{Z} 7$ revealed that 1,331 predicted proteins (representing $11.03 \%$ of the proteome) contain a secretion signal peptide (Armenteros et al. 2019). Secondary metabolites (SMs) play an essential role in the survival of fungi in their ecological niche. Fungal SM-related gene clusters were predicted by the online tool antiSMASH 5.0 (Blin et al. 2019), which identified 31 SM clusters (6 nonribosomal peptides synthetase [NRPS], 9 type 1 [T1] polyketide synthase [PKS], 7 terpene, 7 NRPS-like, 1 fungal-RiPP, and 1 T3 PKS). The R genoplotR package was used to visualize the SM gene clusters in the genome of A. alternata Z7 (Fig. 1).

For comparative genomics analysis, the genomes of $A$. solani CBS_143772 (tomato early blight, GenBank accession GCA_002952155.1), A. gaisen FERA_650 (black spot of Japanese pear, GenBank accession GCA_004156025.2), A. alternata JS-0527 (an endophytic fungus isolated from a leaf tissue of Phragmites australis, GenBank accession GCA_011420255.1), A. tenuissima FERA 1166 (isolated from infected leaves of apple, GenBank accession GCA_004156035.1), and A. alternata NAP07 (isolated from diseased lesions of peach, GenBank accession GCA_009932595.1) were collected and compared with the
A. alternata $\mathrm{Z7}$ (tangerine pathotype) genome using all-vs-all BLAST search with a strict threshold '-evalue 1e-5 -outfmt 6'. Alignments with cumulative sizes smaller than $2 \mathrm{~kb}$ were removed and the filtered alignments were clustered into syntenic blocks. The macrosynteny plots were constructed using the $\mathrm{R}$ genoplotR package (Guy et al. 2010). The result showed remarkably syntenic and collinear relationships among different Alternaria genomes (Fig. 2). Interestingly, we found that A. alternata $\mathrm{Z7}$ is composed of 10 ECs and 2 CDCs (GenBank accession numbers CP061875.1

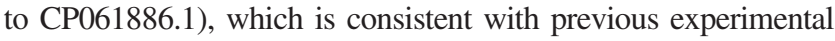
evidence generated by pulsed-field gel electrophoresis (Wang et al. 2019). We also found that most Alternaria spp. have 10 essential chromosomes, although some of these may have undergone rearrangements. Interestingly, we did not find homologs of CDCs in the endophytic fungus $A$. alternata JS-0527, concordant with the fact that CDCs play a vital role in the pathogenicity of $A$. alternata.

To investigate the species similarity of Alternaria, the nucleotidelevel genomic similarity of 27 Alternaria spp. were analyzed by pyANI and visualized by the $\mathrm{R}$ pheatmap package. In the average nucleotide identity (ANI) heatmap, A. alternata strains ATCC11680, ATCC66891, BMP0270, JS-0527, FERA1177, B2a, JS-1623, SRC11rK2f, and Z7 showed more than 98\% ANI (Fig. 3). We found that A. gaisen, also known as A. alternata Japanese pear pathotype, has more than $98 \%$ ANI similarity with $A$. fragariae and $96 \%$ ANI similarity with A. alternata. Furthermore, we found that the ANI of A. tenuissima, A. turkisafria, A. limoniasperae, A. mali, A. citriarbusti, Alternaria sp. MG1, and A. alternata showed more than $98 \%$ similarity, which is congruent with multigene phylogenies based on commonly used gene regions (Woudenberg et al. 2015).

To our knowledge, this is the first report of the chromosomelevel genome assembly of A. alternata using the Illumina and Oxford Nanopore sequencing platforms. The assembly had a total genome size of $34.28 \mathrm{Mb}$, and the contig $\mathrm{N}_{50}$ reached $3.08 \mathrm{Mb}$. In total, 12,067 protein-coding genes were predicted, and 7,907 genes $(65.52 \%)$ have been annotated. Macrosynteny analysis of the chromosome-level genome of Alternaria spp. showed syntenic and collinear relationships among different Alternaria genomes. The genome sequence of $A$. alternata $\mathrm{Z7}$ reported here is a significant improvement over previous genome assembly. We also performed comparative genomics analysis and found highly differentiated genomes among A. alternata strains, which will be valuable in further study of the preferential virulence to its original host plants. The genomic data in this study offer a valuable resource for facilitating the comparative genomics of Alternaria spp. as well as for studying the molecular basis of the pathogenicity of A. alternata.

Table 2. Genomic features of Alternaria alternata Z7 (GenBank accession GCA_014751505.1) ${ }^{\mathrm{a}}$

\begin{tabular}{|c|c|c|c|c|c|c|c|c|}
\hline ID $^{\mathbf{b}}$ & $\begin{array}{l}\text { Genome } \\
\text { size (bp) }\end{array}$ & $\begin{array}{c}\text { GC } \\
\text { content }(\%)\end{array}$ & $\begin{array}{l}\text { Protein-coding } \\
\text { genes }\end{array}$ & $\begin{array}{l}\text { tRNA } \\
\text { genes }^{c}\end{array}$ & $\begin{array}{l}\text { Gene density } \\
\text { (genes/Mb) }\end{array}$ & $\begin{array}{l}\text { Average length } \\
\text { of gene (bp) }\end{array}$ & $\begin{array}{l}\text { Average number } \\
\text { of exons/gene }\end{array}$ & $\begin{array}{l}\text { GenBank } \\
\text { accession }\end{array}$ \\
\hline Chr 1 & $6,770,053$ & 51.19 & 2,446 & 38 & 361 & 1,955 & 2.55 & CP061875.1 \\
\hline Chr 2 & $5,542,836$ & 51.01 & 2,052 & 26 & 370 & 1,882 & 2.47 & CP061876.1 \\
\hline Chr 3 & $3,269,484$ & 51.06 & 1,193 & 22 & 365 & 1,864 & 2.49 & CP061877.1 \\
\hline Chr 4 & $3,085,453$ & 51.04 & 1,124 & 9 & 364 & 1,956 & 2.55 & CP061878.1 \\
\hline Chr 5 & $2,863,349$ & 50.88 & 1,003 & 10 & 350 & 1,960 & 2.59 & CP061879.1 \\
\hline Chr 6 & $2,544,158$ & 50.96 & 877 & 22 & 345 & 1,938 & 2.45 & CP061880.1 \\
\hline Chr 7 & $2,519,108$ & 50.89 & 868 & 16 & 345 & 1,995 & 2.59 & CP061881.1 \\
\hline Chr 8 & $2,464,928$ & 51.06 & 861 & 9 & 349 & 1,843 & 2.45 & CP061882.1 \\
\hline Chr 9 & $2,401,432$ & 50.91 & 820 & 17 & 341 & 2,023 & 2.55 & CP061883.1 \\
\hline Chr 10 & $1,841,737$ & 51 & 628 & 13 & 341 & 1,976 & 2.5 & CP061884.1 \\
\hline CDC 1 & 561,391 & 50.3 & 121 & 0 & 216 & 1,651 & 2.07 & CP061885.1 \\
\hline CDC 2 & 416,412 & 50.4 & 74 & 0 & 178 & 1,663 & 2.27 & CP061886.1 \\
\hline
\end{tabular}

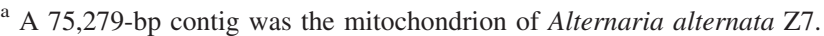

${ }^{\mathrm{b}} \mathrm{Chr}=$ chromosome and $\mathrm{CDC}=$ conditionally dispensable chromosome .

c tRNA $=$ transfer RNA.
} 


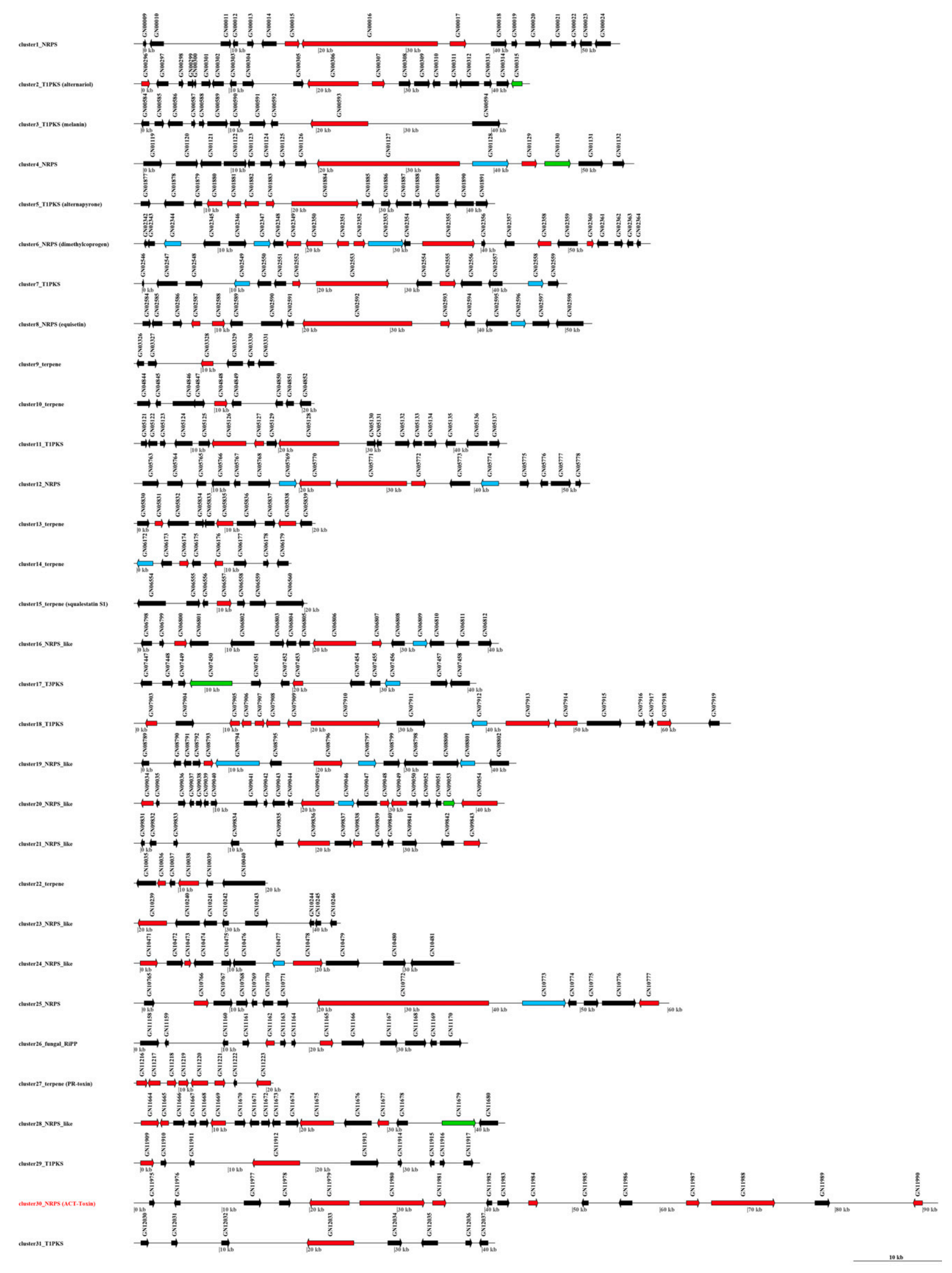

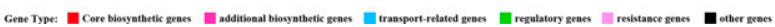

Fig. 1. Secondary metabolite gene clusters of Alternaria alternata Z7. Abbreviations: PKS = polyketide synthase, NRPS = nonribosomal peptides synthetase, $\mathrm{T} 1=$ type 1 , and terpene $=$ terpene synthetase. 


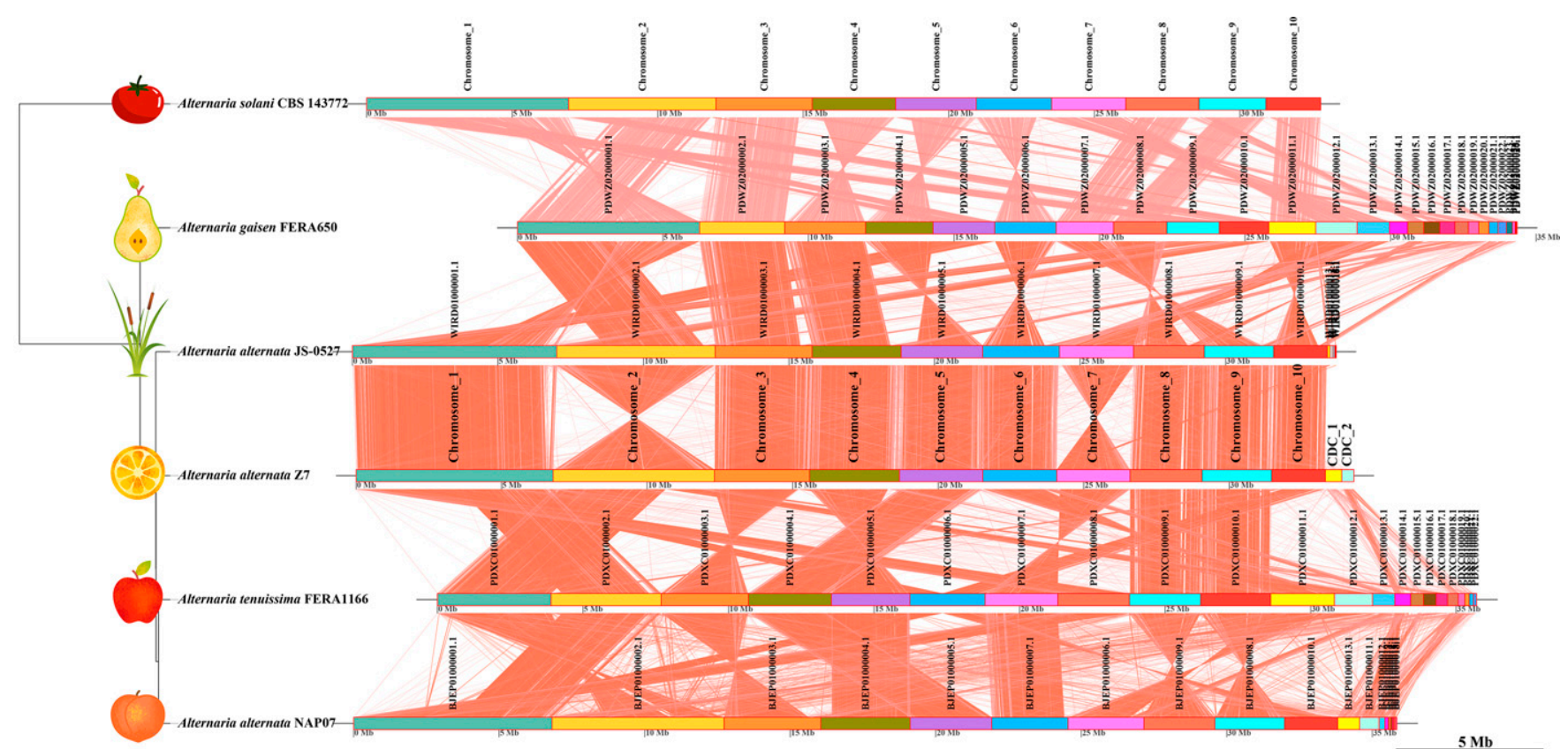

Fig. 2. Synteny and collinearity analysis of genome of Alternaria alternata Z7 (GenBank accession GCA_014751505.1) and five Alternaria genomes using NCBI BLASTN. The phylogenetic tree with genome alignment was generated by the R GenoPlotR package.

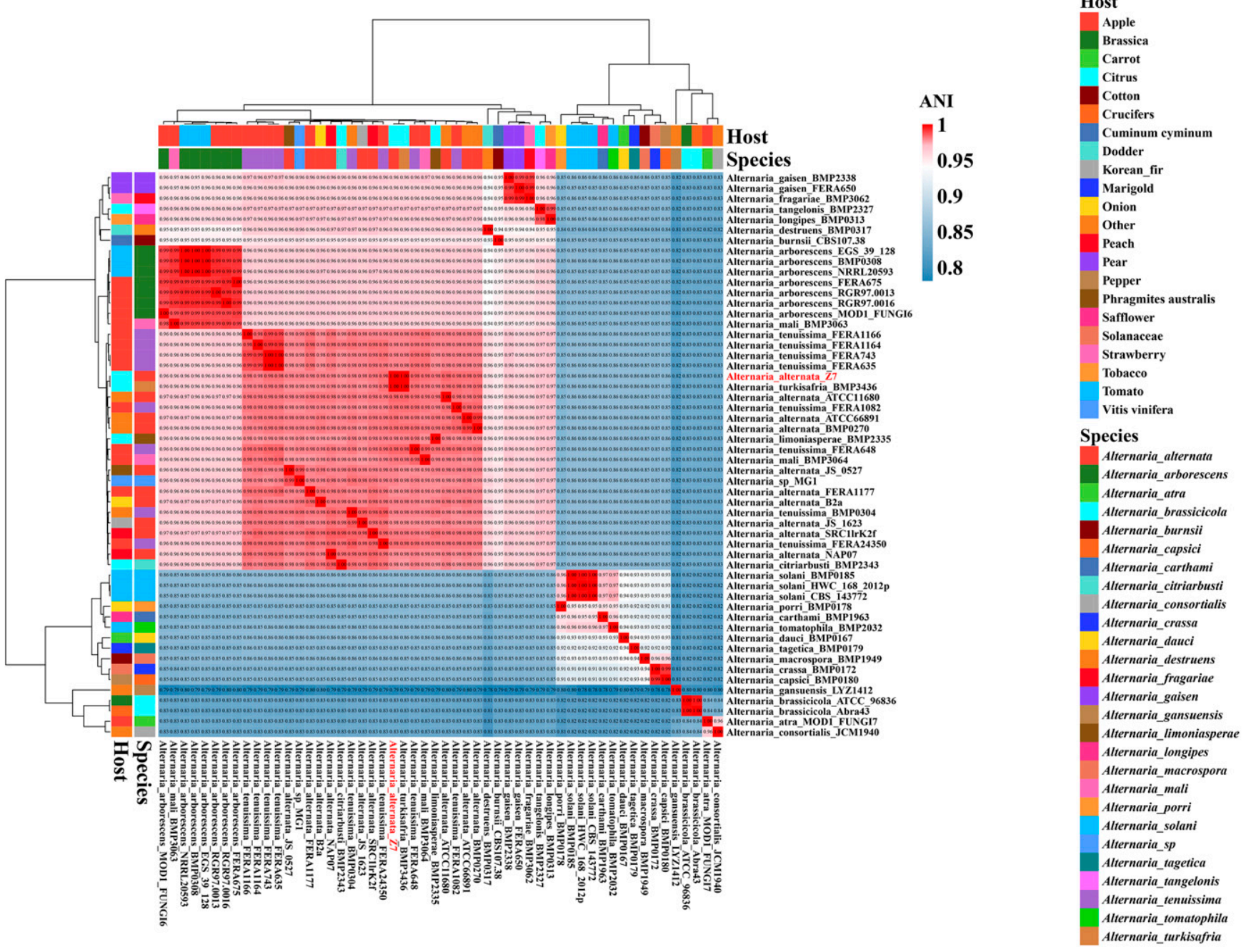

Fig. 3. Heatmap showing the average nucleotide identity (ANI) relationships among 54 Alternaria genomes that span the diversity of different Alternaria spp., including Alternaria alternata. The ANI heatmap was generated by the R Pheatmap package. 


\section{Data availability.}

This Whole-Genome project has been deposited at NCBI BioProject under the accession PRJNA661160. The chromosomal sequence and gene annotation of $\mathrm{Z7}$ can be found at GenBank with accession numbers to GCA_014751505.1. Oxford Nanopore reads, Illumina reads, and RNA-seq Illumina data are available in the NCBI BioSample under the accession numbers SAMN16047574, SAMN16047331, and SAMN15746765, respectively. The wholegenome sequences are available at GenBank under the accession number CP061875.1 for chromosome 1, CP061876.1 for chromosome 2, CP061877.1 for chromosome 3, CP061878.1 for chromosome 4, CP061879.1 for chromosome 5, CP061880.1 for chromosome 6, CP061881.1 for chromosome 7, CP061882.1 for chromosome 8, CP061883.1 for chromosome 9, CP061884.1 for chromosome 10, CP061885.1 for CDC 1, and CP061886.1 for CDC 2. The SM biosynthesis clusters of A. alternata Z7 are available online. The whole-genome and annotation data are also available at the ZJUDATA website.

\section{ACKNOWLEDGMENTS}

We thank D. Cook, S. Moenga, and B. Alford for helpful scientific discussion; H. Fu and F. Huang for technical support and helpful comments on this study; Beijing Biomarker Technologies Corporation for supporting Oxford Nanopore sequencing; and Annoroad Company (Beijing, China) for supporting whole-genome sequencing.

\section{AUTHOR-RECOMMENDED INTERNET RESOURCES}

A. alternata Z7: http://www.zjudata.com/alternaria/Z7/

Alternaria genomes database: http://www.zjudata.com/alternaria/blast.php ANI Calculator: http://enve-omics.ce.gatech.edu/ani/ antiSMSH fungal version: https://fungismash.secondarymetabolites.org/ Benchmarking Universal Single-Copy Orthologs (BUSCO): https://busco.ezlab.org/

Carbohydrate-Active enZYmes Database: http://www.cazy.org/

Citrus Fungal Disease Database: http://www.zjudata.com/

NECAT: https://github.com/xiaochuanle/NECAT

NCBI BioProject accession PRJNA661160:

https://www.ncbi.nlm.nih.gov/bioproject/661160

Pathogen-Host Interaction (PHI) database: http://www.phi-base.org/

pyANI: http://huttonics.github.io/pyani/

RepeatMasker v4.0.5: http://repeatmasker.org/

RepeatModeler v1.0.8: http://www.repeatmasker.org/RepeatModeler/

SignalP-5.0 Server: http://www.cbs.dtu.dk/services/SignalP/

SNAP v2013.02.16: http://ftp.debian.org/debian/pool/main/s/snap/

ZJUDATA website: http://www.zjudata.com/alternaria/blast.php

\section{LITERATURE CITED}

Aiello, D., Guarnaccia, V., Azzaro, A., and Polizzi, G. 2020. Alternaria brown spot on new clones of sweet orange and lemon in Italy. Phytopathol. Mediterr. 59:131-145.

Ajiro, N., Miyamoto, Y., Masunaka, A., Tsuge, T., Yamamoto, M., Ohtani, K., Fukumoto, T., Gomi, K., Peever, T. L., Izumi, Y., Tada, Y., and Akimitsu, K. 2010. Role of the host-selective ACT-toxin synthesis gene ACTTS2 encoding an enoyl-reductase in pathogenicity of the tangerine pathotype of Alternaria alternata. Phytopathology 100:120-126.

Akimitsu, K., Tsuge, T., Kodama, M., Yamamoto, M., and Otani, H. 2014. Alternaria host-selective toxins: Determinant factors of plant disease. J. Gen. Plant Pathol. 80:109-122.

Armenteros, J. J. A., Tsirigos, K. D., Sønderby, C. K., Petersen, T. N., Winther, O., Brunak, S., von Heijne, G., and Nielsen, H. 2019. SignalP 5.0 improves signal peptide predictions using deep neural networks. Nat. Biotechnol. 37:420-423.

Armitage, A. D., Cockerton, H. M., Sreenivasaprasad, S., Woodhall, J., Lane, C. R., Harrison, R. J., and Clarkson, J. P. 2020. Genomics evolutionary history and diagnostics of the Alternaria alternata species group including apple and Asian pear pathotypes. Front. Microbiol. 10:3124.

Bairoch, A., and Apweiler, R. 2000. The SWISS-PROT protein sequence database and its supplement TrEMBL in 2000. Nucleic Acids Res. 28:45-48.

Birney, E., Clamp, M., and Durbin, R. 2004. GeneWise and Genomewise. Genome Res. 14:988-995.
Blin, K., Shaw, S., Steinke, K., Villebro, R., Ziemert, N., Lee, S. Y., Medema, M. H., and Weber, T. 2019. antiSMASH 5.0: Updates to the secondary metabolite genome mining pipeline. Nucleic Acids Res. 47:W81-W87.

Brůna, T., Lomsadze, A., and Borodovsky, M. 2020. GeneMark-EP+: Eukaryotic gene prediction with self-training in the space of genes and proteins. NAR Genomics Bioinf. 1:1qaa026.

Bushmanova, E., Antipov, D., Lapidus, A., and Prjibelski, A. D. 2019. rnaSPAdes: A de novo transcriptome assembler and its application to RNA-Seq data. Gigascience 8:giz100.

Campbell, M. A., Haas, B. J., Hamilton, J. P., Mount, S. M., and Buell, C. R. 2006. Comprehensive analysis of alternative splicing in rice and comparative analyses with Arabidopsis. BMC Genomics 7:327.

Canihos, Y., Erkilic, A., and Timmer, L. 1997. First report of Alternaria brown spot of Minneola tangelo in Turkey. Plant Dis. 81:1214.

Cantarel, B. L., Korf, I., Robb, S. M., Parra, G., Ross, E., Moore, B., Holt, C., Sánchez Alvarado, A., and Yandell, M. 2008. MAKER: An easy-touse annotation pipeline designed for emerging model organism genomes. Genome Res. 18:188-196.

Chung, K.-R. 2012. Stress response and pathogenicity of the necrotrophic fungal pathogen Alternaria alternata. Scientifica (Cairo) 2012:635431.

Cobb, N. 1903. Letters on the disease of plants-Alternaria of the citrus tribe. Agric. Gaz. N.S.W. 14:955-986.

Dang, H. X., Pryor, B., Peever, T., and Lawrence, C. B. 2015. The Alternaria genomes database: A comprehensive resource for a fungal genus comprised of saprophytes, plant pathogens, and allergenic species. BMC Genomics 16:239.

Elena, K. 2006. Alternaria brown spot of Minneola in Greece; evaluation of citrus species susceptibility. Eur. J. Plant Pathol. 115:259-262.

Fu, H., Chung, K. R., Gai, Y., Mao, L., and Li, H. 2020. The basal transcription factor II H subunit $\mathrm{Tfb} 5$ is required for stress response and pathogenicity in the tangerine pathotype of Alternaria alternata. Mol. Plant Pathol. 21:1337-1352.

Gai, Y., Li, L., Ma, H., Riely, B. K., Liu, B., and Li, H. 2021. The critical role of MetR/MetB/MetC/MetX in cysteine and methionine metabolism, fungal development and virulence of Alternaria alternata. Appl. Environ. Microbiol. 87:e01911-20.

Gai, Y., Liu, B., Ma, H., Li, L., Chen, X., Moenga, S., Riely, B., Fayyaz, A., Wang, M., and Li, H. 2019. The methionine biosynthesis regulator AaMetR contributes to oxidative stress tolerance and virulence in Alternaria alternata. Microbiol. Res. 219:94-109.

Grabherr, M. G., Haas, B. J., Yassour, M., Levin, J. Z., Thompson, D. A., Amit, I., Adiconis, X., Fan, L., Raychowdhury, R., Zeng, Q., Chen, Z., Mauceli, E., Hacohen, N., Gnirke, A., Rhind, N., di Palma, F., Birren, B. W., Nusbaum, C., Lindblad-Toh, K., Friedman, N., and Regev, A. 2011. Full-length transcriptome assembly from RNA-Seq data without a reference genome. Nat. Biotechnol. 29:644-652.

Guy, L., Kultima, J. R., and Andersson, S. G. 2010. genoPlotR: Comparative gene and genome visualization in R. Bioinformatics 26:2334-2335.

Haas, B. J., Salzberg, S. L., Zhu, W., Pertea, M., Allen, J. E., Orvis, J., White, O., Buell, C. R., and Wortman, J. R. 2008. Automated eukaryotic gene structure annotation using EVidenceModeler and the Program to Assemble Spliced Alignments. Genome Biol. 9:R7.

Hatta, R., Ito, K., Hosaki, Y., Tanaka, T., Tanaka, A., Yamamoto, M., Akimitsu, K., and Tsuge, T. 2002. A conditionally dispensable chromosome controls host-specific pathogenicity in the fungal plant pathogen Alternaria alternata. Genetics 161:59-70.

Hoff, K. J., and Stanke, M. 2019. Predicting genes in single genomes with AUGUSTUS. Curr. Protoc. Bioinformatics 65:e57.

Huang, F., Fu, Y., Nie, D., Stewart, J. E., Peever, T. L., and Li, H. 2015. Identification of a novel phylogenetic lineage of Alternaria alternata causing citrus brown spot in China. Fungal Biol. 119:320-330.

Kakvan, N., Zamanizadeh, H., Morid, B., Taheri, H., and Hajmansor, S. 2012. Study on pathogenic and genetic diversity of Alternaria alternato isolated from citrus hybrids of Iran, based on RAPD-PCR technique. Eur. J. Exp. Biol. 2:570-576.

Kim, D., Paggi, J. M., Park, C., Bennett, C., and Salzberg, S. L. 2019. Graph-based genome alignment and genotyping with HISAT2 and HISAT-genotype. Nat. Biotechnol. 37:907-915.

Li, H., Handsaker, B., Wysoker, A., Fennell, T., Ruan, J., Homer, N., Marth, G., Abecasis, G., Durbin, R., and 1000 Genome Project Data Processing Subgroup. 2009. The sequence alignment/map format and SAMtools. Bioinformatics 25:2078-2079.

Li, H., Mei, X., Fu, Y., Huang, F., Chen, Z., Yang, X., Wang, Y., and Huang, H. 2015. Alternaria brown spot of citrus: The risk and management strategy. J. Fruit Sci. 32:969-976.

Lombard, V., Golaconda Ramulu, H., Drula, E., Coutinho, P. M., and Henrissat, B. 2014. The carbohydrate-active enzymes database (CAZy) in 2013. Nucleic Acids Res. 42:D490-D495. 
Ma, H., Zhang, B., Gai, Y., Sun, X., Chung, K.-R., and Li, H. 2019. Cellwall-degrading enzymes required for virulence in the host selective toxin-producing necrotroph Alternaria alternata of citrus. Front. Microbiol. 10:2514.

Marín, J. E., Fernández, H. S., Peres, N. A., Andrew, M., Peever, T. L., and Timmer, L. W. 2006. First report of Alternaria brown spot of citrus caused by Alternaria alternata in Peru. Plant Dis. 90:686.

Miyamoto, Y., Masunaka, A., Tsuge, T., Yamamoto, M., Ohtani, K. Fukumoto, T., Gomi, K., Peever, T. L., Tada, Y., Ichimura, K., and Akimitsu, K. 2010. ACTTS3 encoding a polyketide synthase is essential for the biosynthesis of ACT-toxin and pathogenicity in the tangerine pathotype of Alternaria alternata. Mol. Plant-Microbe Interact. 23: 406-414.

Nishimura, S., and Kohmoto, K. 1983. Host-specific toxins and chemical structures from alternaria species. Annu. Rev. Phytopathol. 21: 87-116.

Peres, N. A. R., Agostini, J. P., and Timmer, L. W. 2003. Outbreaks of Alternaria brown spot of citrus in Brazil and Argentina. Plant Dis. 87:750.

Simão, F. A., Waterhouse, R. M., Ioannidis, P., Kriventseva, E. V., and Zdobnov, E. M. 2015. BUSCO: Assessing genome assembly and annotation completeness with single-copy orthologs. Bioinformatics 31: 3210-3212.

Solel, Z. 1991. Alternaria brown spot on Minneola tangelos in Israel. Plant Pathol. 40:145-147.

Swart, S., Wingfield, M., Swart, W., and Schutte, G. 1998. Chemical control of Alternaria brown spot on Minneola tangelo in South Africa. Ann. Appl. Biol. 133:17-30.

Thomma, B. P. 2003. Alternaria spp.: From general saprophyte to specific parasite. Mol. Plant Pathol. 4:225-236.

Tsuge, T., Harimoto, Y., Akimitsu, K., Ohtani, K., Kodama, M., Akagi, Y., Egusa, M., Yamamoto, M., and Otani, H. 2013. Host-selective toxins produced by the plant pathogenic fungus Alternaria alternata. FEMS Microbiol. Rev. 37:44-66.

Vicent, A., Armengol, J., Sales, R., García-Jiménez, J., and Alfaro-Lassala, F. 2000. First report of Alternaria brown spot of citrus in Spain. Plant Dis. 84:1044.

Walker, B. J., Abeel, T., Shea, T., Priest, M., Abouelliel, A., Sakthikumar, S., Cuomo, C. A., Zeng, Q., Wortman, J., Young, S. K., and Earl, A. M. 2014. Pilon: An integrated tool for comprehensive microbial variant detection and genome assembly improvement. PLoS One 9:e112963.

Wang, M., Fu, H., Shen, X. X., Ruan, R., Rokas, A., and Li, H. 2019. Genomic features and evolution of the conditionally dispensable chromosome in the tangerine pathotype of Alternaria alternata. Mol. Plant Pathol. 20: $1425-1438$

Wang, M., Sun, X., Yu, D., Xu, J., Chung, K., and Li, H. 2016. Genomic and transcriptomic analyses of the tangerine pathotype of Alternaria alternata in response to oxidative stress. Sci. Rep. 6:32437.

Wang, M., Yang, X., Ruan, R., Fu, H., and Li, H. 2018. Csn5 is required for the conidiogenesis and pathogenesis of the Alternaria alternata tangerine pathotype. Front. Microbiol. 9:508.

Wang, X. F., Li, Z. A., Tang, K. Z., Zhou, C. Y., and Yi, L. 2010. First report of Alternaria brown spot of citrus caused by Alternaria alternata in Yunnan Province, China. Plant Dis. 94:375.

Whiteside, J. 1976. A newly recorded Alternaria-induced brown spot disease on Dancy tangerines in Florida. Plant Dis. Rep. 3:326-329.

Winnenburg, R., Baldwin, T. K., Urban, M., Rawlings, C., Köhler, J., and Hammond-Kosack, K. E. 2006. PHI-base: A new database for pathogen host interactions. Nucleic Acids Res. 34:D459-D464.

Woudenberg, J. H., Seidl, M. F., Groenewald, J. Z., de Vries, M., Stielow, J. B., Thomma, B. P., and Crous, P. W. 2015. Alternaria section Alternaria: Species, formae speciales or pathotypes? Stud. Mycol. 82: $1-21$. 